\title{
TUBERCULOSE PULMONAR: ACHADOS TOMOGRÁFICOS EM PACIENTES COM DOENÇA PULMONAR EM ATIVIDADE NO HOSPITAL UNIVERSITÁRIO DA UNIVERSIDADE FEDERAL DO PIAUÍ (HU-UFPI)
}

\author{
PULMONARY TUBERCULOSIS: TOMOGRAPHIC FINDIGNS IN PATIENTS WITH ACTIVE PULMONARY \\ DISEASE AT THE UNIVERSITY HOSPITAL OF THE PIAUÍ FEDERAL UNIVERSITY (HU-UFPI)
}

Lorena Lima Bezerra', Daniel Lopes da Cunha ${ }^{2}$

${ }^{1}$ Residência Médica em Radiologia e Diagnóstico por Imagem, Hospital Universitário da Universidade Federal do Piauí (HU/UFPI), Teresina, Piauí, Brasil. Email: lorenamufpi@gmail.com

2 Departamento de Radiologia e Diagnóstico por Imagem do Hospital Universitário da Universidade Federal do Piauí (HU/UFPI), Teresina, Piauí, Brasil. Especialização em Diagnóstico por Imagem do Sistema Músculo, Faculdade da Grande Fortaleza, Fortaleza, Ceará. Brasil. E-mail: danlopescunha@gmail.com

\section{RESUMO}

INTRODUÇÃO: A tuberculose é uma doença infectocontagiosa que configura um grave problema de saúde pública mundial. O diagnóstico de atividade de doença em pacientes com tuberculose pulmonar depende de múltiplos fatores. A tomografia computadorizada, ao localizar a doença ao nível do lóbulo pulmonar secundário, fornece informações adicionais, sendo útil na instituição do tratamento precoce. OBJETIVOS: Descrever os achados tomográficos em pacientes com tuberculose pulmonar em atividade acompanhados no Hospital Universitário da Universidade Federal do Piauí no período de 2016 a 2019. Procurou-se discutir a importância da tomografia no diagnóstico da atividade de doença em pacientes com comprovação bacteriológica ou forte suspeita clínica e avaliar a concordância interobservador. METODOLOGIA: Estudo transversal, descritivo, com coleta retrospectiva dos dados. Variáveis: presença e distribuição pulmonar dos achados tomográficos. Para a análise foram obtidas médias, porcentagens e utilizados testes Qui-quadrado e Kappa. RESULTADOS: A maioria dos pacientes eram do sexo masculino (72,5\%); a idade na época do diagnóstico variou entre 20 anos e 81 anos. A baciloscopia foi positiva em $42,5 \%$ dos casos. Os achados tomográficos mais frequentes foram: nódulos centrolobulares $(72,5 \%)$, espessamento parietal brônquico $(55,0 \%)$ e aspecto de 'árvore em brotamento' (52,5\%). CONCLUSÃO: A tomografia computadorizada se mostra de extrema importância na avaliação de sinais precoces de disseminação broncogênica do bacilo de Koch, sendo útil na instituição do tratamento precoce, particularmente naquelas pacientes com forte suspeita clínica e baciloscopia negativa. Houve concordância do ponto de vista estatístico na avaliação interobservador da maioria dos achados.

DESCRITORES: Tuberculose pulmonar. Tomografia computadorizada. 


\section{ABSTRACT}

INTRODUCTION: Tuberculosis is an infectious disease that is a serious worldwide public health problem. The diagnosis of disease activity in patients with pulmonary tuberculosis depends on multiple factors. Computed tomography, by locating the disease at the level of the secondary pulmonary lobe, provides additional information and is useful in instituting early treatment. OBJECTIVES: To describe the tomographic findings in patients with active pulmonary tuberculosis followed at the University Hospital of the Federal University of Piauí from 2016 to 2019. We sought to discuss the importance of tomography in the diagnosis of disease activity in patients with bacteriological confirmation or strong clinical suspcion of tuberculosis, and assess interobserver agreement. METHODOLOGY: Cross-sectional, descriptive study with retrospective data collection. Variables: presence and pulmonary distribution of tomographic findings. For the analysis, means, percentages were obtained and Chisquare and Kappa tests were used. RESULTS: Most patients were male (72.5\%); Age at diagnosis ranged from 20 to 81 years. The smear was positive in $42.5 \%$ of the cases. The most frequent tomographic findings were: centrilobular nodules (72.5\%), bronchial parietal thickening (55.0\%) and 'tree in bud' appearance (52.5\%). CONCLUSION: Computed tomography is extremely important in the evaluation of early signs of bronchogenic dissemination of the Koch bacillus, being useful in the institution of early treatment, particularly in those patients with strong clinical suspicion and negative smear. There was statistical agreement in the interobserver evaluation of most findings.

KEYWORDS: Tuberculosis Pulmonary. Tomography, X-ray computed.

\section{Como citar este artigo (Vancouver):}

Bezerra LL, Cunha DL. Tuberculose pulmonar: achados tomográficos em pacientes com doença pulmonar em atividade no Hospital Universitário da Universidade Federal do Piauí (HU-UFPI). J. Ciênc. Saúde [internet]. 2019;2(3):23-37. Disponível em: https://doi.org/10.26694/jcs hu-ufpi.v2i3.11895 


\section{INTRODUÇÃO}

A tuberculose (TB) é uma doença infectocontagiosa bacteriana, transmissível quase exclusivamente por aerossol, causada pelo Mycobacterium tuberculosis ou Bacilo de Koch (BK). Afeta principalmente os pulmões (tuberculose pulmonar) e pode também acometer outros órgãos (tuberculose extrapulmonar) ou, ainda, ocorrer de forma disseminada ${ }^{1}$.

É um grave problema de saúde pública mundial e milhares de pessoas ainda adoecem e morrem devido à doença e suas complicações. É a nona principal causa de morte no mundo e principal causa de um único agente infeccioso, acima de HIV / AIDS. Em 2016, havia uma estimativa de 1,3 milhões de mortes por TB entre pessoas HIV negativas ${ }^{2}$.

No Brasil, em 2017, foram notificados 69.569 casos novos de tuberculose. Nesse mesmo ano, o coeficiente de incidência foi igual a 33,5 casos/100 mil habitantes. No período de 2008 a 2017, esse coeficiente apresentou queda média anual de 1,6\%. Em 2016, foram registrados 4.426 óbitos por tuberculose, resultando em um coeficiente de mortalidade igual a 2,1 óbitos/100 mil habitantes, que apresentou queda média anual de 2,0\% de 2007 a $2016^{3}$.

Em 2017 a distribuição dos indicadores foi heterogênea nas regiões do Brasil, com coeficiente de incidência variando de 20 casos/100 mil habitantes (Centro-Oeste) a 42,7 casos/100 mil habitantes (Norte). Na região nordeste a incidência foi de 31,2 casos $/ 100$ mil habitantes, com número de casos novos notificados de 17.869 casos $^{3}$.

No estado do Piauí no ano de 2017, o número de casos novos notificados foi de 622 , o coeficiente de incidência da tuberculose foi de 19,3 /100 mil habitantes e o coeficiente de mortalidade por TB em 2016 foi de 2,5 / 100 mil hab. Em Teresina, capital do Piauí, no ano de 2017 houve 237 casos novos de TB notificados, com coeficiente de incidência da tuberculose de 27,9 /100 mil habitantes e coeficiente de mortalidade por TB em 2016 foi de 3,3 / 100 mil habitantes $^{3}$.
A transmissão da tuberculose ocorre quando o organismo é expelido pela tosse, espirro ou fala de pacientes infectados. Embora a TB possa afetar qualquer órgão, a maioria das infecções em pacientes imunocompetentes é restrita aos pulmões ${ }^{4,5}$.

A doença pulmonar pode ser classificada como primária ou secundária. Os sintomas primários da tuberculose são geralmente leves e os pacientes são relativamente assintomáticos. A doença secundária ocorre principalmente devido a reativação endógena da tuberculose latente, com sintomas como tosse, dor torácica, fraqueza ou cansaço, perda de peso, febre e sudorese noturna ${ }^{4-6}$.

O diagnóstico de atividade de doença em pacientes com tuberculose pulmonar depende de múltiplos fatores, como quadro clínico, exame físico, teste tuberculínico e, principalmente, a detecção do bacilo no escarro, no lavado broncoalveolar, em aspirado transtraqueal ou em fragmentos de tecido pulmonar obtidos por biópsia. $\mathrm{O}$ diagnóstico definitivo se dá pela identificação dos BKs em amostra biológica através da baciloscopia, da cultura ou de métodos moleculares (PCR $)^{4,5,8}$.

A baciloscopia ou pesquisa de BAAR (bacilo álcool-ácido resistente) é o método mais utilizado por ser rápido e de baixo custo. Devido a sua baixa sensibilidade, deve ser realizada no mínimo em duas amostras. Desde que executada corretamente em todas as suas fases, a baciloscopia permite detectar de $60 \%$ a $80 \%$ dos casos de TB pulmonar. Contudo, por ser totalmente realizada pelo profissional do laboratório, não havendo automatização, é dependente do profissional executante. Sua sensibilidade também varia de acordo com o tipo de lesão, o tipo e o número de amostras. A sensibilidade da técnica também é prejudicada em pacientes paucibacilares ${ }^{2,6,9}$.

O exame de cultura permite $\mathrm{O}$ isolamento $\mathrm{e}$ crescimento de BAAR em meios específicos. É considerado o exame padrão-ouro, com alta sensibilidade e especificidade para a detecção de TB pulmonar e extrapulmonar ${ }^{8}$. É mais sensível que a baciloscopia, promovendo um incremento no diagnóstico de cerca de $20 \%$. A cultura pela metodologia tradicional, em meio sólido, manual, pode 
levar até sessenta dias para liberação de um resultado. Os métodos automatizados em meio líquido podem ter resultados em até 14 dias, mas tem alto custo ${ }^{6}$.

A radiografia do tórax continua sendo a primeira escolha na avaliação inicial de pacientes com tuberculose pulmonar. Apesar de a radiografia de tórax poder oferecer informações sobre a atividade da doença, algumas alterações parenquimatosas mínimas podem não ser identificadas, principalmente em estágios iniciais da doença ${ }^{10,11}$. A tomografia computadorizada multidetectores (TCMD) mostrou-se superior à radiografia simples na avaliação da presença e extensão das alterações parenquimatosas, já que permite a localização da doença ao nível do lóbulo pulmonar secundário ${ }^{4,12,13}$.

A tomografia computadoriza do tórax com múltiplos detectores é uma ferramenta importante na detecção de doença pulmonar oculta radiograficamente. Proporciona um melhor diagnóstico diferencial das lesões parenquimatosas pulmonares, melhor avaliação de linfonodos mediastinais e avaliação da atividade da doença e de suas complicações, permitindo um diagnóstico mais precoce e preciso das lesões pulmonares. A TCMD permite avaliar bronquiectasias, cavitações, bolas fúngicas e avaliação adicional de necrose dos linfonodos mediastinais, identificando patologias pleurais, de vias aéreas e diafragmáticas, além de avaliar as estruturas ósseas da caixa torácica ${ }^{10,11}$.

A TCMD adiciona informações no diagnóstico e é um método particularmente valioso para os pacientes com suspeita de TB e com baciloscopia negativa, permitindo isolamento destes pacientes e adequada terapêutica empírica enquanto se espera o resultado da cultura de escarro para confirmação de $\mathrm{TB}^{10}$.

Pacientes com baciloscopia negativa têm um risco menor de transmitir a doença do que os pacientes com baciloscopia positiva, mas ainda são capazes de transmitir a doença. A taxa de transmissão relativa de pacientes não bacilíferos em comparação com os bacilíferos foi verificada em $22 \%$ utilizando um método epidemiológico molecular. Considerando-se que grande parte dos pacientes (quase 50\%) com TB pode ter resultados negativos no esfregaço de escarro, a contribuição total desses pacientes na transmissão da doença se torna notável ${ }^{13}$.

Como resultado, os médicos frequentemente hesitam em iniciar o tratamento por medo de possíveis efeitos colaterais dos medicamentos antituberculose. Nessas situações, a TCAR (Tomografia Computadorizada de Alta Resolução) pode ajudar no diagnóstico provisório da tuberculose, para que a terapia empírica possa ser iniciada e, por outro lado, selecionar pacientes que provavelmente não têm tuberculose ${ }^{10,13}$.

Os achados tomográficos mais comuns da TB pulmonar em atividade são os micronódulos centrolobulares, opacidade em 'árvore em brotamento', cavitação e consolidação. Estes achados estão intimamente relacionados com o número de BAAR encontrados na baciloscopia, possibilitando avaliação da infectividade em pacientes com TB pulmonar ${ }^{6,9,14}$.

Para a identificação precoce dos casos de $\mathrm{TB}$, um rápido e eficaz diagnóstico se faz necessário. $O$ diagnóstico de atividade pode ser difícil, tendo em vista que baciloscopia de escarro pode ser negativa em 21 a 66\% dos casos, a cultura pode levar até seis semanas para o crescimento de colônias e os achados nas radiografias de tórax são frequentemente indeterminados ${ }^{4,5}$. A incapacidade de diagnosticar e tratar rapidamente os pacientes afetados leva ao aumento da morbimortalidade, desenvolvimento de resistência secundária e transmissão contínua da doença. Um melhor entendimento da contribuição de cada um dos principais métodos de diagnósticos e a relação entre eles se faz necessária.

O objetivo primário deste estudo foi descrever os principais achados tomográficos em aparelho com multidetectores (TCMD) em pacientes com suspeita de tuberculose pulmonar em atividade acompanhados no Hospital Universitário da Universidade Federal do Piauí (HU-UFPI) e discutir a importância da tomografia computadorizada no diagnóstico da tuberculose pulmonar em atividade em pacientes com comprovação bacteriológica ou forte suspeita clínica. Como objetivos 
secundários, procurou-se comparar os resultados encontrados com os achados descritos na literatura e avaliar a concordância interobservador na análise tomográfica em pacientes com tuberculose pulmonar em atividade, bem como sua reprodutibilidade no nosso meio.

\section{METODOLOGIA}

Trata-se de um estudo observacional, transversal e descritivo, com coleta retrospectiva dos dados, realizado no Hospital Universitário da Universidade Federal do Piauí (HU-UFPI), serviço que atende totalmente pelo Sistema Único de Saúde (SUS). As informações dos pacientes com tuberculose pulmonar em atividade foram obtidas a partir dos registros nos cadernos de notificação compulsória da vigilância epidemiológica do HU-UFPI, com posterior análise dos dados nos seus respectivos prontuários eletrônicos do AGHU (Aplicativo de Gestão para Hospitais Universitários). A população foi composta por pacientes com tuberculose pulmonar acompanhados no ambulatório de pneumologia e pacientes internados no HU-UFPI no período de janeiro de 2016 a agosto de 2019 , os quais preencheram os seguintes critérios de inclusão: diagnóstico bacteriológico confirmado de tuberculose pulmonar ou forte suspeita clínica; ter sido paciente do ambulatório de pneumologia do HUUFPI; ter realizado tomografia computadorizada no Hospital Universitário da Universidade Federal do Piauí (HUUFPI). Foram excluídos aqueles pacientes com tuberculose extrapulmonar, com micobacteriose não tuberculosa, que não possuíam estudo tomográfico realizado no HU-UFPI ou que já haviam realizado tratamento prévio para tuberculose pulmonar.

Para o cálculo de amostra representativa da população geral, utilizou-se a seguinte fórmula para análise de amostras finitas ${ }^{16}$.

$n=N \times \delta^{2} \times(Z \alpha / 2)^{2} /(N-1) \times\left(E^{2}\right)+\delta^{2} \times(Z \alpha / 2)^{2}$
Onde $\mathbf{N}$ representa o tamanho da amostra, $\boldsymbol{\delta}$ o desvio padrão populacional da variável (presença de alterações tomográficas centrolobulares em pacientes com tuberculose pulmonar em atividade), $\mathbf{Z} \alpha$ o valor crítico para o grau de confiança, que equivale a 1,96 (95\%); E o erro padrão (5\%). Para um $\delta$ estimado em 97\%, segundo Campos et al. temos uma amostra representativa de 39 pacientes ${ }^{12}$.

Foi preenchida uma ficha de coleta de dados elaborada pelos autores da pesquisa, avaliando-se a presença e distribuição pulmonar dos seguintes achados: pequenos nódulos pulmonares (dimensões inferiores a $3 \mathrm{~mm}$ ) e seu padrão de distribuição (centrolobular, perilinfático ou miliar), aspecto de 'árvore em brotamento', espessamento parietal brônquico, espessamento septal, cavitação, consolidação, opacidades em 'vidro-fosco', massa pulmonar, linfonodomegalias mediastinais (diâmetro do menor eixo superior a $10 \mathrm{~mm}$ ) e derrame pleural.

As imagens tomográficas dos pacientes inclusos na pesquisa, disponíveis no sistema de armazenamento de imagens do HU-UFPI, foram reavaliadas de forma independente por médico radiologista com 9 anos de experiência, orientador da pesquisa, e pela autora do projeto, residente do terceiro em Radiologia e Diagnóstico por Imagem, em estação de trabalho Siemens com ferramentas de reconstrução multiplanar.

Os dados obtidos foram tabelados e digitados em planilhas no Microsoft Excel for Windows, transferidos e analisados com o programa Statistical Package for the Social Science (SPSS) versão 20.0. Na análise univariada utilizaram-se tabelas de frequência, gráficos, medidas de posição e dispersão ${ }^{17}$. Para análise bivariada, utilizou-se o Teste Qui-quadrado de Pearson $\left(\mathrm{X}^{2}\right)$ para associar as variáveis qualitativas ou classificações, quando atendidas as pressuposições do teste. Foi aplicado o Teste de Kappa para avaliar a concordância interobservador na análise tomográfica em pacientes com tuberculose pulmonar em atividade. Neste estudo foi adotado $5 \%(p<0,05)$ como nível de significância estatística, valor para o qual houve concordância na avaliação interobservador do ponto de vista estatístico ${ }^{17-19}$. 
A coleta de dados iniciou-se somente após a aprovação do trabalho pelo Comitê de Ética em Pesquisa do HUUFPI (CEP HU-UFPI), de acordo com a Resolução $n^{\circ} 466$ de 2012 do Conselho Nacional de Saúde. O trabalho foi aprovado pelo CEP HU-UFPI sob CAAE 13331019.1.0000.8050 e número do parecer 3.375.036. Os pacientes envolvidos na pesquisa foram contactados por telefone e por via direta e pessoal pela assistente da pesquisa, com gastos custeados pela mesma, sendo feita a solicitação de permissão para a utilização dos dados no presente estudo. Nos casos em que não foi possível contato direto ou por telefone, foi solicitado dispensa do termo de consentimento livre e esclarecido.

\section{RESULTADOS}

No presente estudo, foram analisados os casos de 40 pacientes com diagnóstico bacteriológico ou forte suspeita clínica de tuberculose pulmonar. A maioria dos pacientes eram do sexo masculino (72,5\%); o percentual do sexo feminino foi de $27,5 \%$. A idade dos pacientes na época do diagnóstico da doença variou entre 20 anos e 81 anos. A média de idade foi de 51 anos, com desvio padrão de 17,2 anos (TABELA 1).

Tabela 1 - Distribuição do sexo e idade dos pacientes com tuberculose pulmonar em atividade acompanhados no HU-UFPI durante o período de 2016 e 2019. Teresina, 2019.

\begin{tabular}{llll}
\hline Variáveis & $\mathbf{N}$ & $\%$ & Estatísticas \\
\hline Sexo & 11 & 27,5 & - \\
Feminino & 29 & 72,5 & - \\
Masculino & 40 & 100,0 & - \\
Total & 40 & 100,0 & Min=20; Max $=81 ; \mathrm{M}=51 ;$ \\
\hline Idade & & & DP $=17,2$
\end{tabular}

Legenda: Min= idade mínima. Max= idade máxima. $\mathrm{M}=$ média das idades. $\mathrm{DP}=$ Desvio padrão. $\mathrm{N}=$ quantidade de pacientes da amostra. Fonte: Hospital Universitário - HU-UFPI, 2019

Em relação ao quadro clínico à época do exame tomográfico, a tosse foi o sintoma mais frequente, sendo referida por 27 pacientes (67,5\%). A febre e perda ponderal foram outras manifestações clínicas frequentes, sendo referidas por $17(42,5 \%)$ e 16 pacientes $(40 \%)$, respectivamente. 13 pacientes $(32,5 \%)$ referiram dispneia, 7 pacientes $(17,5 \%)$ referiram astenia e dor torácica; 5 pacientes $(12,5)$ apresentaram escarros hemoptoicos (TABELA 2).
Outros sintomas menos frequentes relatados foram: cervicalgia, lombalgia, tumoração em hemitórax, poliatralgia, disfagia e edema. Houve um caso assintomático; tratava-se de um paciente com doença de Crohn cuja suspeição diagnóstica foi feita após alteração de exame de PPD na avaliação pré-terapia com imunobiológico. 
Tabela 2 - Distribuição dos achados clínicos em pacientes com tuberculose pulmonar no HU-UFPI durante o período de 2016 e 2019. Teresina, 2019.

\begin{tabular}{llc}
\hline Achados clínicos & $\mathbf{N}$ & $\mathbf{\%}$ \\
\hline TOSSE & 27 & 67,5 \\
FEBRE & 17 & 42,5 \\
PERDA PONDERAL & 16 & 40,0 \\
DISPNEIA & 13 & 32,5 \\
ASTENIA & 7 & 17,5 \\
DOR TORÁCICA & 7 & 17,5
\end{tabular}

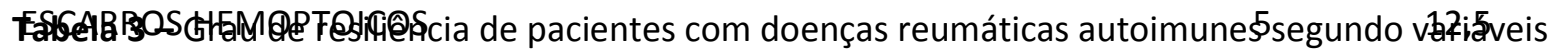
sociodemneráficas. Teresina/PI. 2019 (continuacão) Legenda: $\mathrm{N}=$ quantidade de pacientes da amostra. Fonte: Hospital Universitário - HU-UFPI.

O diagnóstico de tuberculose pulmonar foi feito através de baciloscopia positiva em 17 pacientes (42,5\%). Dezessete $(42,5 \%)$ dos 40 pacientes apresentaram baciloscopia negativa, seja no escarro espontâneo, escarro induzido ou no lavado broncoalveolar. Em 15\% dos casos (6 pacientes), a baciloscopia não foi realizada ou o resultado não estava disponível nos registros eletrônicos (GRÁFICO 1).

Gráfico 1 - Baciloscopia em pacientes com tuberculose pulmonar no HU-UFPI durante o período de 2016 e 2019.

Teresina, 2019.

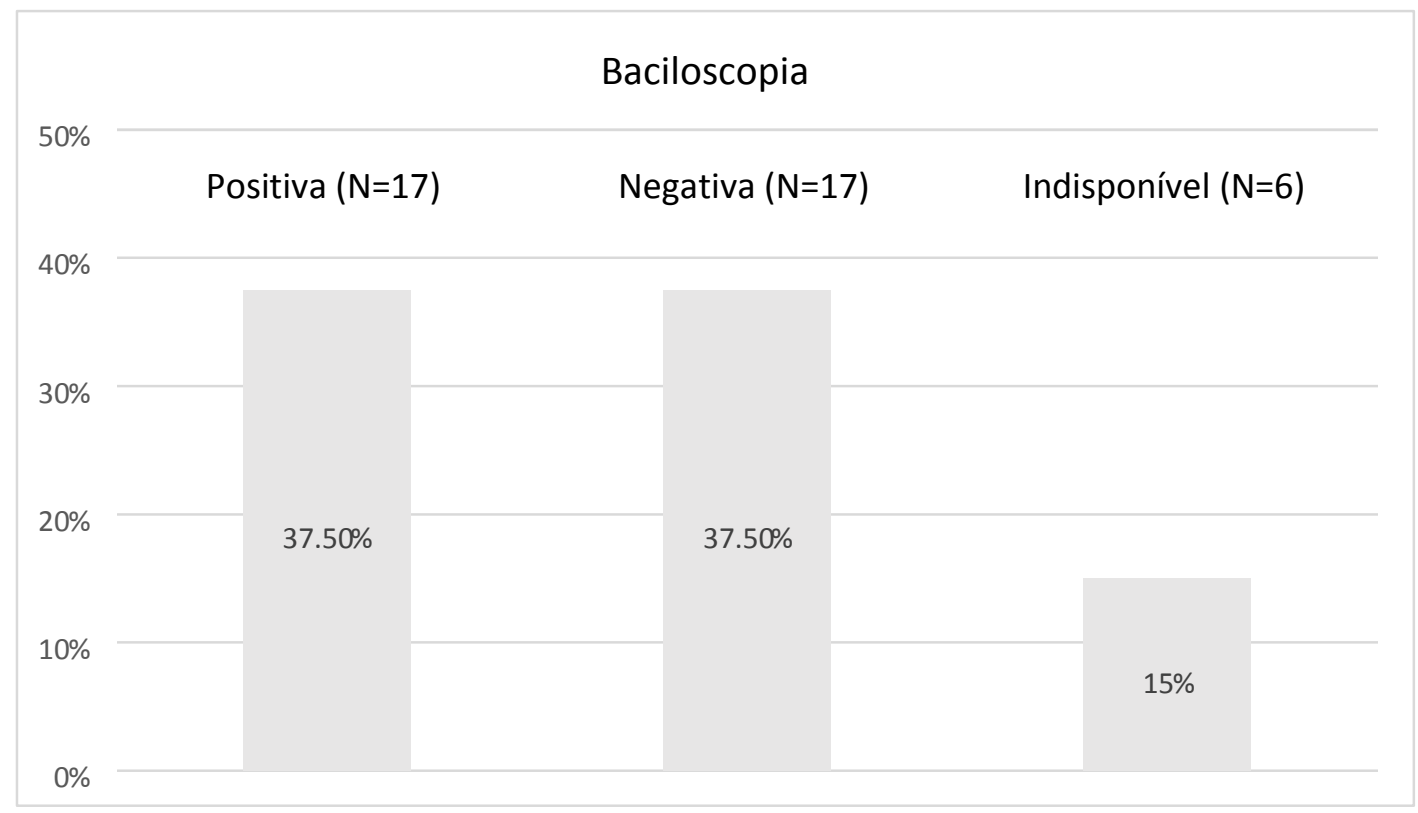

Legenda: N= quantidade de pacientes da amostra. Fonte: Hospital Universitário - HU-UFPI. 
O teste rápido molecular (TRM) foi positivo em 15 resultado não estava disponível nos registros pacientes (37,5\%) e negativo em 14 pacientes (35\%). Em eletrônicos (TABELA 3).

11 pacientes (27,5\%), o exame não foi realizado ou o

Tabela 3 - Resultado do teste rápido molecular em pacientes com tuberculose pulmonar no HU-UFPI durante o período de 2016 e 2019. Teresina, 2019.

\begin{tabular}{lll}
\hline TESTE RÁPIDO MOLECULAR (TRM) & $\mathbf{N}$ & $\mathbf{\%}$ \\
\hline POSITIVO & 15 & 37,5 \\
NEGATIVO & 14 & 35 \\
INDISPONÍVEL & 11 & $\mathbf{2 7 , 5}$ \\
\hline Total & $\mathbf{4 0}$ & $\mathbf{1 0 0 , 0}$ \\
\hline
\end{tabular}

Legenda: $\mathrm{N}=$ quantidade de pacientes da amostra. Fonte: Hospital Universitário - HU-UFPI

Em 17,5\% (7 pacientes) dos casos, tanto baciloscopia, TRM e cultura resultaram negativos; instituiu-se tratamento empírico com base nos dados clínicos e achados tomográficos.

Tabela 4 - Comorbidades em pacientes com tuberculose pulmonar no HU-UFPI durante o período de 2016 e 2019. Teresina, 2019.

\begin{tabular}{lll}
\hline Variáveis & $\mathbf{N}$ & $\mathbf{\%}$ \\
\hline COMORBIDADES & 26 & 65,0 \\
Sim & 14 & 35,0 \\
Não & & \\
DESCRIÇÃO DAS COMORBIDADES (N=26) & 5 & 19,2 \\
DOENÇA DE CROHN & 3 & 11,5 \\
ESPONDILODISCITE & 3 & 11,5 \\
ARTRITE REUMATOIDE & 15 & 57,7 \\
Outras & & \\
\hline
\end{tabular}

Legenda: $\mathrm{N}=$ quantidade de pacientes da amostra. Fonte: Hospital Universitário - HU-UFPI

$\mathrm{Na}$ avaliação das tomografias computadorizadas de tórax, observou-se que $82,5 \%$ delas apresentavam pequenos nódulos pulmonares, sendo a localização centrolobular mais frequente, vista em $72,5 \%$ das vezes.
Comorbidades diversas estavam presentes em até 26 dos 40 pacientes (65\%), sendo a mais frequente Doença de Crohn, vista em 19,2\% dos casos (TABELA 4). 
centrolobulares assumiram aspecto de 'árvore em brotamento' em 21 pacientes (52,5\%).

Tabela 5 - Distribuição dos pequenos nódulos pulmonares em pacientes com tuberculose pulmonar no HU-UFPI durante o período de 2016 e 2019. Teresina, 2019.

\begin{tabular}{|c|c|c|c|c|}
\hline \multirow{2}{*}{ Variável } & \multicolumn{2}{|c|}{ Grau de Resiliência } & \multirow[b]{2}{*}{ Total } & \multirow[b]{2}{*}{ P-Valoı } \\
\hline & Baixo & Moderado/Elevado & & \\
\hline \multicolumn{5}{|l|}{ Atividade da doença } \\
\hline Remissão/leve & $2(14,3)$ & $12(85,7)$ & 14 & \multirow{2}{*}{$0,157^{3}$} \\
\hline Moderada/alta/muito alta & $10(38,5)$ & $16(61,5)$ & 26 & \\
\hline \multicolumn{5}{|l|}{ Uso de corticoides } \\
\hline Sim & $4(28,6)$ & $10(71,4)$ & 14 & \multirow{2}{*}{$1,000^{3}$} \\
\hline Não & $8(26,7)$ & $22(73,3)$ & 30 & \\
\hline \multicolumn{5}{|l|}{ Dose de Prednisona } \\
\hline$>10 \mathrm{mcg} / \mathrm{dl}$ & $1(14,3)$ & $6(85,7)$ & 7 & \multirow{2}{*}{$0,653^{3}$} \\
\hline$<=10 \mathrm{mcg} / \mathrm{dl}$ & $11(29,7)$ & $26(70,3)$ & 37 & \\
\hline \multirow{2}{*}{ Tempo de diagnóstico } & $7,8 \pm 8,21^{\mathrm{M}}$ & $6,8 \pm 6,08^{M}$ & - & \multirow{2}{*}{$0,864^{5}$} \\
\hline & $5,5 \pm 8^{\mathrm{D}}$ & $6,0 \pm 6,79^{D}$ & - & \\
\hline
\end{tabular}

Legenda: $\mathrm{N}=$ quantidade de pacientes da amostra. Fonte: Hospital Universitário - HU-UFPI.

Houve distorção da arquitetura pulmonar habitual em 17 pacientes (42,5\%); espessamento parietal brônquico foi caracterizado em 22 casos (55\%).

Cavitações, linfonodomegalias mediastinais, derrame pleural e opacidades em 'vidro-fosco' foram caracterizadas em 16 pacientes cada (40\%). Consolidação foi vista em 13 pacientes (32,5\%). Os nódulos do espaço aéreo foram encontrados em 12 pacientes (30\%), enquanto espessamento septal foi visto em $25 \%$ das vezes. Massa pulmonar ocorreu em 5 pacientes $(12,5 \%)$ (TABELA 6).

Tabela 6 - Frequência de achados tomográficos em pacientes com tuberculose pulmonar no HU-UFPI durante o período de 2016 e 2019. Teresina, 2019.

\begin{tabular}{lll}
\hline Achados tomográficos & N & \% \\
\hline Pequenos nódulos pulmonares & 33 & 82,5 \\
Espessamento parietal brônquico & 22 & 55,0 \\
Aspecto de "árvore em brotamento" & 21 & 52,5 \\
Cavitação & 16 & 40,0 \\
Linfonodomegalia mediastinal & 16 & 40,0 \\
Derrame pleural & 16 & 40,0 \\
Opacidades em "vidro-fosco" & 16 & 40,0 \\
Consolidação & 13 & 32,5 \\
Massa pulmonar & 5 & 12,5
\end{tabular}

Legenda: $\mathrm{N}=$ quantidade de pacientes da amostra. Fonte: Hospital Universitário - HU-UFPI. 
Considerando-se os achados tomográficos apenas no subgrupo de pacientes com baciloscopia negativa (17 pacientes), observamos menor incidência absoluta da maioria dos achados tomográficos àqueles com baciloscopia positiva (TABELA 7).
Micronódulos centrolobulares foram vistos em 14 pacientes com baciloscopia positiva $(82,3 \%)$ e 10 pacientes $(58,8 \%)$ com baciloscopia negativa.

Tabela 7 - Frequência de achados tomográficos em pacientes com tuberculose pulmonar e baciloscopia negativa e positiva no HU-UFPI durante o período de 2016 e 2019. Teresina, 2019.

\begin{tabular}{lllll}
\hline Achados tomográficos & $\mathbf{N}=\mathbf{B N}$ & $\mathbf{\%}$ & $\mathbf{N}=\mathbf{B P}$ & $\%$ \\
\hline Pequenos nódulos pulmonares & 12 & 70,5 & 15 & 88,2 \\
Espessamento parietal brônquico & 8 & 47,0 & 11 & 64,0 \\
Aspecto de "árvore em brotamento" & 6 & 35,3 & 12 & 70,5 \\
Cavitação & 5 & 29,4 & 9 & 52,9 \\
Linfonodomegalia mediastinal & 3 & 17,6 & 11 & 64,0 \\
Derrame pleural & 5 & 29,4 & 10 & 58,8 \\
Opacidades em "vidro-fosco" & 6 & 35,3 & 8 & 47,0 \\
Consolidação & 3 & 17,6 & 9 & 52,9 \\
Massa pulmonar & 3 & 17,6 & 3 & 17,6
\end{tabular}

Legenda: $\mathrm{N}=$ quantidade de pacientes da amostra; $\mathrm{BN}=$ baciloscopia negativa; $\mathrm{BP}=$ baciloscopia positiva. Fonte: Hospital Universitário - HU-UFPI.

Ao se avaliar a concordância interobservador na análise tomográfica em pacientes com tuberculose pulmonar em atividade feita de forma independente por médico radiologista com 9 anos de experiência e por residente do terceiro ano em Radiologia e Diagnóstico por Imagem, observou-se associação positiva, significativa do ponto de vista estatístico $(p<0,05)$ para a maioria dos achados, excetuando-se nódulos perilinfáticos, opacidade em 'vidro-fosco' e espessamento septal.

\section{DSICUSÃO}

No presente estudo, 17 pacientes (42,5\%) apresentaram baciloscopia positiva e 17 pacientes $(42,5 \%)$ apresentaram baciloscopia negativa, fato condizente com os dados da literatura, em que nas diversas casuísticas observam um percentual de baciloscopias negativas em pacientes com doença em atividade variando de 21 a $47 \%{ }^{20,21}$.
Das diversas comorbidades presentes, a mais frequente foi a Doença de Crohn, vista em 19,2\% dos casos (TABELA 4), achado que pode ser justificado pela situação do HU-UFPI como referência no tratamento de doenças inflamatórias intestinais.

O conhecimento da fisiopatologia da tuberculose pulmonar se faz necessário para o adequado entendimento das alterações tomográficas características. A forma mais comum de disseminação na tuberculose pós-primária é a endobrônquica. Os achados de disseminação endobrônquica são nódulos centrolobulares, padrão 'árvore em brotamento', espessamento parietal brônquico e consolidação lobular. A doença desenvolve-se inicialmente nos bronquíolos respiratórios, seguida de inflamação local, que se reflete em pequenos nódulos no estudo radiológico. Os micronódulos consistem de material sólido caseoso dentro ou na periferia dos bronquíolos terminais ou respiratórios e alvéolos 
peribronquiolares ${ }^{21,22}$. Micronódulos não são visíveis na radiografia de tórax convencional, e a detecção destes focos discretos na tomografia é altamente sugestiva de tuberculose em atividade (23).

O padrão tomográfico de 'árvore em brotamento' foi inicialmente descrito para caracterizar a aparência de disseminação endobrônquica do $M$. tuberculosis. Representa uma forma de impactação mucoide na qual estruturas lineares possuem mais de uma ramificação. É um achado característico, mas não patognomônico de tuberculose ativa. No contexto clínico adequado, é um critério confiável de atividade de doença ${ }^{22}$.

$\mathrm{Na}$ nossa casuística, os sinais de disseminação broncogênica (micronódulos centrolobulares e o aspecto de 'árvore em brotamento') foram observados em $72,5 \%$ e $52,5 \%$ dos casos, respectivamente, frequências um pouco menores do que as relatadas nos trabalhos de Im et al. e Hatipoglu et al., que observaram esses achados em $91-97 \%$ e $71-72 \%$ dos casos, respectivamente. Ambos os estudos apresentaram delineamentos diferentes, naquele, apenas pacientes com baciloscopia positiva foram estudados; neste, $53 \%$ dos pacientes apresentavam baciloscopia positiva ${ }^{20,22}$.

Nos estudos de Raghuvanshi et al. e Alsowey et al., que avaliaram os achados tomográficos em pacientes com tuberculose pulmonar em atividade e baciloscopias negativas, as frequências encontradas de micronódulos centrolobulares e aspecto de "árvore em brotamento" variaram de $85,4 \%$ - $90 \%$ e 33,33 - 65,8\%, respectivamente, valores mais próximos ao subgrupo de pacientes com baciloscopia negativa do presente estudo, cujas frequências foram $58,8 \%$ e $35,3 \%{ }^{24,25}$.

Im et al., além das alterações já descritas, destacaram o espessamento de paredes brônquicas durante a disseminação broncogênica, que no presente estudo foi o segundo achado mais frequente, caracterizado em $55 \%$ dos pacientes, frequência esta semelhante à relatada pela literatura, que é de 44 a $79 \%{ }^{20}$. O padrão de distribuição miliar foi visto em $5 \%$ das vezes, condizente com os dados da literatura. Tuberculose miliar ocorre em 2 a $6 \%$ dos casos de tuberculose primária, sendo também vista na tuberculose pósprimária quando o mecanismo de defesa do hospedeiro encontra-se deficiente. A aparência tomográfica inclui tanto nódulos circunscritos quando nódulos mal delimitados medindo de 1 a $2 \mathrm{~mm}$, disseminados amplamente pelos pulmões ${ }^{22}$.

A disseminação linfática na tuberculose desempenha um papel importante, particularmente em estágios iniciais e na infecção primária. Um achado tomográfico que corrobora a disseminação linfática da tuberculose é a linfadenopatia. A micobactéria atinge os nódulos e regiões subpleurais através dos linfáticos pulmonares. A incidência da disseminação linfática na tuberculose pósprimária é provavelmente baixa devido à imunidade adquirida. Contudo, existem poucos estudos na literatura sobre os achados tomográficos de disseminação linfática em adultos ${ }^{21,23}$. Por definição, micronódulos peribroncovasculares, septais e subpleurais indicam doença linfática. Suspeita-se que esses micronódulos representem granulomas no interstício pulmonar, o que não é incomum histopatologicamente ${ }^{21-23}$. Ko et al. observaram uma prevalência de micronódulos perilinfáticos de $58 \%$ em pacientes com tuberculose pulmonar em atividade ${ }^{27,28}$. $\mathrm{Na}$ nossa casuística, o padrão de distribuição perilinfático foi visto em $12,5 \%$ dos casos. Acredita-se que micronódulos perilinfáticos em adultos com tuberculose pulmonar ativa tenham sido historicamente negligenciados ou interpretados como nódulos centrolobulares com opacidades ramificadas lineares, o que justifica a baixa frequência deste achado em diversas casuísticas ${ }^{21,27,28}$.

Consolidação e cavitação representam invasão local pelo bacilo de Koch. Poey et al. consideraram a cavitação como o sinal mais importante de atividade de doença ${ }^{29}$; este achado foi visto em $40 \%$ dos pacientes no presente estudo. A consolidação do parênquima pulmonar foi encontrada em $32,5 \%$ dos pacientes. Choi et al. verificaram frequência maior de consolidação, de $73 \%$, em estudo realizado com pacientes graves cursando com quadro de insuficiência respiratória aguda, condições em que os achados clínicos e radiológicos são mais exuberantes ${ }^{30}$.

Doença pleural é mais frequentemente associada à tuberculose primária, mas também ocorre na doença pós-primária. Envolvimento pleural pode ser visto em 
até $38 \%$ dos casos de tuberculose primária e $18 \%$ dos casos de pós-primária. Efusões pleurais uni ou bilaterais foram caracterizadas em $40 \%$ dos $\operatorname{casos}^{31}$.

A opacidade em 'vidro-fosco' foi encontrada em $40 \%$ dos casos estudados, condizente com a frequência relatada na literatura, que é de $35-38 \%{ }^{20-23}$. Linfadenopatia hilar ou mediastinal é vista em $96 \%$ das crianças e $43 \%$ dos adultos com tuberculose pósprimária. A prevalência de adenopatia decresce com a idade. $\mathrm{O}$ aumento de nódulos linfonodais ocorreu em $40 \%$ na nossa casuística, valor próximo à frequência relatada na literatura, que varia de 16 a $31 \%^{18-21}$.

Em alguns casos a tuberculose pulmonar se manifesta como massa mal delimitada, simulando câncer de pulmão primário. Histopatologicamente, a lesão representa um agregado de granulomas com fibrose circunjacente $e^{22,32}$. No nosso estudo, isso foi observado em $12,5 \%$ dos pacientes.

$\mathrm{Na}$ análise comparativa entre a frequência de achados tomográficos nos grupos com baciloscopia negativa e positiva, observamos maior incidência absoluta da maioria dos achados tomográficos em pacientes com baciloscopia positiva (TABELA 7), à exceção das massas pulmonares, cujas frequências foram idênticas nos dois subgrupos avaliados. Discrepância maior ocorreu para o aspecto de "árvore em brotamento", cavitação e consolidação, significativamente maiores no subgrupo com baciloscopia positiva.

Matsuoka et al. investigaram a relação entre os achados da TC em pacientes com tuberculose pulmonar ativa e o valor da baciloscopia nos esfregaços de escarro e descobriram que a frequência dos micronódulos e nódulos não diferiu significativamente entre os grupos com esfregaço positivo e negativo. A frequência de micronódulos pulmonares encontrada por Matsouka et. al. foi de $88 \%$ em pacientes com baciloscopia negativa e $94,6 \%$ em pacientes com baciloscopia positiva; na nossa casuística as frequências foram $70,5 \%$ e $88,2 \%$, respectivamente ${ }^{34}$.

Por outro lado, Matsouka et al. observaram que à medida que o número de BAAR aumenta, consolidação e cavitação se tornam significativamente mais frequentes na TC (34). Nossos resultados estão de acordo com estes achados, sugerindo que a existência de consolidação do espaço aéreo ou cavitação na TC pode indicar alta infectividade, visto que a contagem bacteriana da baciloscopia indica o grau de infectividade e a gravidade da doença.

Embora os micronódulos centrolobulares tenham sido relatados como um importante sinal de tuberculose pulmonar ativa, não observamos diferença significativa na frequência dessa característica entre os dois grupos deste estudo. Consideramos que, embora as opacidades centrolobulares possam refletir uma condição patológica específica, a área total afetada é menor do que na consolidação do espaço aéreo. Portanto, a quantidade de exsudação rica em bacilos e material necrótico também pode ser menor quando comparada à consolidação do espaço aéreo. Além disso, a distância da área afetada às vias aéreas centrais na opacidade centrolobular é maior do que na consolidação e cavitação do espaço aéreo ${ }^{34,35}$.

Houve concordância do ponto de vista estatístico na avaliação interobservador da maioria dos achados tomográficos, com exceção dos nódulos perilinfáticos, opacidade em 'vidro-fosco' e espessamento septal, possibilitando a reprodutibilidade do método no nosso meio.

\section{CONCLUSÃO}

Micronódulos pulmonares, espessamento parietal brônquico e padrão de 'árvore em brotamento' foram as características tomográficas mais frequentes em pacientes com diagnóstico de tuberculose pulmonar em atividade, achados condizentes com a literatura atual. Houve concordância do ponto de vista estatístico na avaliação interobservador da maioria dos achados tomográficos. 


\section{REFERÊNCIAS}

1. Harisinghani M, McLoud T, Shepard J, Ko J, Shroff M, Mueller P. Tuberculosis from Head to Toe.

RadioGraphics. [internet] 2000;20(2):449-70.

Disponível em:

https://doi.org/10.1148/radiographics.20.2.g00mc124 $\underline{49}$

2. Global tuberculosis report 2018. Geneva: World Health Organization; 2018

3. Ministério da Saúde (BR). Secretaria de vigilância em saúde Departamento de Vigilância das Doenças Transmissíveis. Implantação do Plano Nacional pelo Fim da Tuberculose como Problema de Saúde Pública no Brasil: primeiros passos rumo ao alcance das metas. Brasília: Ministério da Saúde; 2018.

4. Dheda K, Barry C, Maartens G. Tuberculosis. The Lancet. [internet] 2016;387(10024):12111226.

Disponível em: https://doi.org/10.1016/s0140-

6736(15)00151-8

5. Oliphant C. Tuberculosis. The Journal for Nurse Practitioners [internet] 2015;11(1):87-94. Disponível em: https://doi.org/10.1016/j.nurpra.2014.10.018

6. Ministério da Saúde (BR). Secretaria de vigilância em saúde. Plano nacional pelo fim da tuberculose como problema de saúde pública. $1^{\circ}$ ed. Brasília: Ministério da Saúde, 2017.

7. Lee K, Hwang J, Chung M, Kim H, Kwon O. Utility of $\mathrm{CT}$ in the Evaluation of Pulmonary Tuberculosis in Patients Without AIDS. Chest. [internet] 1996;110(4):977-84. Disponível em: https://doi.org/10.1378/chest.110.4.977

8. Osório Ferri A, Aguiar B, Mörschbächer Wilhelm C, Schmidt D, Fussieger F, Ulrich Picoli S. Diagnóstico da tuberculose: uma revisão. Revista Liberato [internet] 2014;15(24):145-54. Disponível em:

http://revista.liberato.com.br/ojs lib/index.php/revist a/article/view/317/219

9. Singhal R, Myneedu V. Microscopy as a diagnostic tool in pulmonary tuberculosis. Int J Mycobacteriol. [internet] 2015;4(1):1-6. Disponível em: https://www.ijmyco.org/text.asp?2015/4/1/1/200583

10. Matsuoka S, Uchiyama K, Shima H, Suzuki K, Shimura A, Sasaki Y et al. Relationship between CT findings of pulmonary tuberculosis and the number of acid-fast bacilli on sputum smears. Clinical Imaging. [Internet]. 2004;28(2):119-23. Disponível em: https://doi.org/10.1016/s0899-7071(03)00148-7

\section{Shaarrawy H, Zeidan M, Nasr A, Nouh M.}

Assessment of the role of high resolution computed tomography in the diagnosis of suspected sputum smear negative active pulmonary TB. Egyptian Journal of Chest Diseases and Tuberculosis [Internet]. 2013;62(2):263-8. Disponível em: https://doi.org/10.1016/j.ejcdt.2013.05.006

12. Aló CC, Edson M, Rosana R. Tuberculose pulmonar: achados na tomografia computadorizada de alta resolução do tórax em pacientes com doença em atividade comprovada bacteriologicamente. J. Pneumologia [Internet]. 2002 Jan [citado 17 out. 2019] ; 28(1): 23-29. Disponível em: http://dx.doi.org/10.1590/S010235862002000100006.

13. Kanaya A, Glidden D, Chambers H. Identifying Pulmonary Tuberculosis in Patients With Negative Sputum Smear Results. Chest. 2001;120(2):349-55. Disponível em:

https://doi.org/10.1378/chest.120.2.349

14.Kim H, Song K, Goo J, Lee J, Lee K, Lim T. Thoracic Sequelae and Complications of Tuberculosis. RadioGraphics. 2001;21(4):839-58. Disponível em: https://pubs.rsna.org/doi/pdf/10.1148/radiographics.2 1.4.g01jl06839

15. Ko J, Park H, Kim C, Song S. The relation between CT findings and sputum microbiology studies in active pulmonary tuberculosis. European Journal of Radiology. 2015;84(11):2339-44. Disponível em: http://doi.org/10.1016/j.ejrad.2015.07.032

16. Miot HA. Tamanho da amostra em estudos clínicos e experimentais. J. vasc. bras. 2011;10(4):275-8. Disponível em: https://doi.org/10.1590/S1677$\underline{54492011000400001}$ 
17. Pestana MH, Gageiro JN. Análise de dados para ciência sociais: a complementaridade do SPSS. 3 ed. Lisboa: Edições Sílabo, 2003.

18. Armitage P, Berry G, Matthews JNS. Statistical methods in medical research. 3nd ed. London (GB): Blackwell Scientific Publications; 2002.

19. Hosmer DW, Lemeshow S. Applied Logistic Regression. New York: Wiley, 2000.

20. Hatipoglu O, Osma E, Manisali M, Ucan E, Balci P, Akkoclu $A$ et al. High resolution computed tomographic findings in pulmonary tuberculosis. Thorax [internet]. 1996;51(4):397-402. Disponível em:

https://dx.doi.org/10.1136\%2Fthx.51.4.397

21. Itoh $\mathrm{H}$, Tokunaga $\mathrm{S}$, Asamoto $\mathrm{H}$, Furuta $\mathrm{M}$, Funamoto $\mathrm{Y}$, Kitaichi $\mathrm{M}$ et al. Radiologic-pathologic correlations of small lung nodules with special reference to peribronchiolar nodules. AJR Am J Roentgenol [internet] 1978;130(2):2232-31. Disponível em: https://doi.org/10.2214/ajr.130.2.223

22. Im JG, Itoh H, Shim Y, Lee J, Ahn J, Han M et al. Pulmonary tuberculosis: CT findings--early active disease and sequential change with antituberculous therapy. Radiology. 1993;186(3):653-60. Disponível em: https://doi.org/10.1148/radiology.186.3.8430169

23. Lee $\mathrm{K}, \mathrm{Im} \mathrm{J.} \mathrm{CT} \mathrm{in} \mathrm{adults} \mathrm{with} \mathrm{tuberculosis} \mathrm{of} \mathrm{the}$ chest: characteristic findings and role in management. American Journal of Roentgenology [internet]. 1995;164(6):1361-7. Disponível em: https://doi.org/10.2214/air.164.6.7754873

24. Raghuvanshi V, Gopal Sood R, Jhobta A, Sarkar M, Tomar A, Khanna S. Use of High-Resolution Computed Tomography (HRCT) in Diagnosis of Sputum Negative Pulmonary Tuberculosis. Turkish Thoracic Journal [internet]. 2016;17(2):59-64.22. Disponível em: https://dx.doi.org/10.5578\%2Fttj.17.2.012

25. Alsowey A, Amin M, Said A. The Predictive Value of Multidetector High Resolution Computed Tomography in Evaluation of Suspected Sputum Smear Negative Active Pulmonary Tuberculosis in Egyptian Zagazig
University Hospital Patients. Pol J Radiol. [internet]. 2017;82:808-16. Disponível em: https://dx.doi.org/10.12659\%2FPJR.903743

26. Fujita J, Bandoh S, Kubo A, Ishii T, Kanaji, N, Nakamura, H. HRCT shows variations in appearance in disseminated tuberculosis in adults. Int J Tuberc Lung Dis. [internet]. 2006; 10:222-6. Disponível em: https://pubmed.ncbi.nlm.nih.gov/16499265/

27. Ko J, Park H, Kim C. Clinicoradiologic Evidence of Pulmonary Lymphatic Spread in Adult Patients With Tuberculosis. American Journal of Roentgenology. [internet]. 2015;204(1):38-43. Disponível em: https://dx.doi.org/10.2214/AJR.14.12908

28. Ko J, Park H, Kim C, Song S. The relation between $\mathrm{CT}$ findings and sputum microbiology studies in active pulmonary tuberculosis. European Journal of Radiology. [internet]. 2015;84(11):2339-44. Disponível em: http://doi.org/10.1016/j.ejrad.2015.07.032

29. Poey C, Verhaegen F, Giron J, Lavayssiere J, Fajadet P, Duparc B. High Resolution Chest CT in Tuberculosis: Evolutive Patterns and Signs of Activity. J Comput Assist Tomogr. [internet]. 1997;21(4):601-7. Disponível em: https://doi.org/10.1097/00004728-199707000$\underline{00014}$

30. Choi D, Lee K, Suh G, Kim T, Kwon O, Rhee C et al. Pulmonary Tuberculosis Presenting as Acute Respiratory Failure: Radiologic Findings. Journal of Computer Assisted Tomography [internet]. 1999;23(1):107-13. Disponível em: https://doi.org/10.1097/00004728-199901000-00023

31. Curvo-Semedo L, Teixeira L, Caseiro-Alves F. Tuberculosis of the chest. European Journal of Radiology. [internet]. 2005;55(2):158-72. Disponível em: https://doi.org/10.1016/j.ejrad.2005.04.014

32. Lee J, Chong P, Lin C, Hsu A, Lee C. High resolution chest $\mathrm{CT}$ in patients with pulmonary tuberculosis:

Characteristic findings before and after antituberculous therapy. European Journal of Radiology. [internet]. 2008;67(1):100-4. Disponível em: https://doi.org/10.1016/i.eirad.2007.07.009 
33. Lee J, Lee K, Jung K, Han J, Kwon O, Kim J et al. Pulmonary Tuberculosis: $\mathrm{CT}$ and Pathologic Correlation. Journal of Computer Assisted Tomography [internet]. 2000;24(5):691-8. Disponível em: https://doi.org/10.1097/00004728-200009000-00005

34. Matsuoka S, Uchiyama K, Shima H, Suzuki K, Shimura A, Sasaki Y et al. Relationship between CT findings of pulmonary tuberculosis and the number of acid-fast bacilli on sputum smears. Clinical Imaging. [internet]. 2004;28(2):119-23. Disponível em:https://doi.org/10.1016/S0899-7071(03)00148-7
35. Kosaka N, Sakai T, Uematsu H, Kimura H, Hase M, Noguchi $\mathrm{M}$ et al. Specific High-Resolution Computed Tomography Findings Associated With Sputum SmearPositive Pulmonary Tuberculosis. Journal of Computer Assisted Tomography [internet]. 2005;29(6):801-4. Disponível em:

https://doi.org/10.1097/01.rct.0000184642.19421.a9

Fontes de financiamento: Não

Conflito de interesse: Não

Aceito: 18/03/2020

Publicação: 27/04/2020

Endereço para correspondência: Lorena Lima Bezerra. Teresina, Piauí, Brasil. E-mail: lorenamufpi@gmail.com 